\title{
Sickle Hepatopathy and Complex Jaundice in a Primigravida with Hepatitis B Virus Infection: Case Report
}

\author{
Jesse A. Otegbayo ${ }^{1 *}$, Daniel D. Ogbaro ${ }^{2}$, Bamikole T. Osibowale ${ }^{1}$, Olufemi O. Sanyaolu ${ }^{1}$ \\ ${ }^{1}$ Department of Internal Medicine, Babcock University/Babcock University Teaching Hospital, Ilishan-Remo, \\ Nigeria \\ ${ }^{2}$ Department of Haematology, Babcock University/Babcock University Teaching Hospital, llishan-Remo, Nigeria \\ Email: ${ }^{*}$ otegbayoj@babcock.edu.ng
}

Received 16 August 2015; accepted 15 September 2015; published 18 September 2015

Copyright (c) 2015 by authors and Scientific Research Publishing Inc.

This work is licensed under the Creative Commons Attribution International License (CC BY). http://creativecommons.org/licenses/by/4.0/

c) (i) Open Access

\begin{abstract}
Background Aim: This article aimed to sensitize the healthcare providers who care for patients with sickle cell disease (SCD) during pregnancy, to the multiple causes of jaundice in sickle hepatopathy, and sift the maze in establishing the dominant cause. Case Presentation: This is a case of a 28-yr-old Gravida 1 Para $0+0$ health worker, with sickle cell anaemia and background history of peptic ulcer disease, total right hip replacement and previous multiple blood transfusions due to haemolytic crisis. She presented with upper abdominal pain and progressively deepening jaundice. There was minimal relief of the abdominal pain with anti-ulcer medications. She had tender hepatomegaly, positive Murphy's sign and deranged liver function parameters. She was also positive for viral hepatitis B markers. A remarkable finding on abdominal ultrasonography was that of a gallbladder with normal wall thickness and multiple stones. She was managed conservatively on intravenous fluids, antibiotics and Livolin forte (Phosphatidylcholine) with improved liver function parameters within a week. Conclusion: Since sickle cell hepatopathy is multifactorial, the importance of unravelling the dominant cause of jaundice or liver dysfunction in these patients and the need for immediate intervention are necessary for effective and targeted care, hence this case report. Our patient had background chronic hepatitis $B$ with a superadded acute cholecystitis with non-obstructing gallstones, and possible subsequent bacterial hepatitis, which responded to antibiotics. The most likely dominant cause of jaundice was chronic hepatitis $B$ that was precipitated by acute cholecystitis.
\end{abstract}

\section{Keywords}

Sickle Cell Anaemia, Sickle Hepatopathy, Jaundice, Pregnancy

\footnotetext{
${ }^{*}$ Corresponding author.

How to cite this paper: Otegbayo, J.A., Ogbaro, D.D., Osibowale, B.T. and Sanyaolu, O.O. (2015) Sickle Hepatopathy and Complex Jaundice in a Primigravida with Hepatitis B Virus Infection: Case Report. Case Reports in Clinical Medicine, 4, 313316. http://dx.doi.org/10.4236/crcm.2015.49062
} 


\section{Introduction}

Alongside countries like Cameroon, Republic of Congo, Gabon and Ghana, the WHO estimates that the prevalence of sickle cell disease (SCD) in Nigeria is between $20 \%$ and $30 \%$, and it constitutes a major factor in morbidity and mortality among the sufferers [1]. Several major acute and chronic complications present as crises and debilitating conditions as a result of recurrent haemolysis and vaso-occlusive episodes, which include bone pains, anaemia, organ infarction, acute chest syndrome, cholecystolithiasis, acute and chronic hepatitis among others.

Most patients with sickle cell anaemia (SCA) tend to have some degree of scleral jaundice even during the steady state due to the usual ongoing and persistent haemolysis. Sickle cell hepatopathy denotes a range of hepatic pathology arising from a wide variety of insults to the liver in patients with SCD [2]. Its occurrence, known to be mainly in the homozygous S state and less in AS, SC and S beta-Thalassaemia, is usually due to vascular obstructive effect of sickled red cells, organ ischaemia and infarction, haemolysis, pigment stone formation, cholestasis and effects of multiple blood transfusions such as viral hepatitis (B and C) and iron overload [2].

In pregnancy, causes of jaundice may predate pregnancy, while some are well known to be associated with cyesis. Liver disease and jaundice occurring during pregnancy may be pregnancy-related such as hyperemesis gravidarum, intrahepatic cholestasis of pregnancy, pre-eclampsia and eclampsia, Haemolysis-Elevated liver enzymes and Low platelets (HELLP) syndrome and acute fatty liver of pregnancy, or pregnancy-unrelated. Pregnancy-unrelated liver diseases are usually pre-existing liver diseases, such as liver cirrhosis and portal hypertension, chronic hepatitis B and C, autoimmune liver disease and Wilson's disease [3]. The third category is liver diseases co-incident with pregnancy, such as viral hepatitis, biliary disease, Budd-Chiari syndrome and druginduced hepatotoxicity.

The occurrence of these liver diseases and jaundice in particular often contribute to morbidity and mortality of the fetus [4], and the SCD patient [5]. Although, multiple complications [6] and hepatobiliary changes in SCD [7] have been published in Nigeria, there is no publication to date on multiple causes of jaundice in SCD and sickle cell hepatopathy. We here present a pregnant woman with SCD, who presented with overlapping causes of jaundice in early pregnancy, to sensitize the healthcare community about the intricacies of sickle hepatopathy with intertwined aetiology of jaundice.

\section{Case Report}

IA, a 28-year-old G1Para $0+0$ healthcare worker, who is a known homozygous haemoglobin S (HbSS) patient, was admitted with a two-day history of upper abdominal pain and progressively deepening jaundice. Abdominal pain was located around the epigastrium, described as gripping and colicky, of gradual onset but progressively worsened, radiated to the upper back and worse with meals. There was minimal relief with antacids and antiulcer medications.

She had associated nausea, but no vomiting or change in bowel habits or pale stools. She had no fever or body pains. She denied history of dark coloured urine, melaena, melaenemesis, hematochezia or hematemesis. She had been a previous recipient of multiple blood transfusions due to haemolytic crises.

She neither smoked cigarette nor imbibed alcohol but had a background history of peptic ulcer disease (PUD), diagnosed on clinical grounds. Three years earlier, she had total right hip replacement due to avascular necrosis of the femoral head. Her mother was diabetic. She reported her steady state packed cell volume (PCV) as $26 \%$.

Clinical examination on admission revealed a woman in painful distress, conscious, pale, icteric, afebrile (axillary temperature $-36.4^{\circ} \mathrm{C}$ ), acyanosed, well hydrated, with no asterixis or peripheral stigmata of chronic liver disease. There was no peripheral oedema.

The abdomen was flat, moved with respiration, there was tender hepatomegaly and a positive Murphy sign. The spleen and kidneys were not palpably enlarged and bowel sounds were normoactive. The rest of the clinical examination was unremarkable.

Laboratory investigations revealed a total FBC of $6300 / \mathrm{mm}^{3}$, PCV-23.2\%, platelet count $-159,000 / \mathrm{mm}^{3}$. Serum electrolytes, urea and creatinine were normal $(\mathrm{Na}-146 \mathrm{mmol} / \mathrm{l}, \mathrm{K}-4.1 \mathrm{mmol} / \mathrm{l}$, Bicarbonate-25 $\mathrm{mmol} / \mathrm{l}$, Urea—21 mg/dl, Cr—0.7 mg/dl). Hepatitis B surface antigen (HBsAg) was positive, anti-HCV negative. Other hepatitis B viral markers were HBV DNA $1350 \mathrm{iu} / \mathrm{ml}$, HBeAg negative, HBeAb positive, HBc IgM negative. Prothrombin time (PT) was 21 s (control—17 s), PTTK 52 s (control—49 s), INR 1.23. Liver Function Test (LFTs) showed aspartate transaminase (AST) - 126 iu/l, alanine transaminase (ALT) $269 \mathrm{iu} / \mathrm{l}$, alkaline phospha- 
tase (ALP) $132 \mathrm{iu} / \mathrm{l}$, Total bilirubin—5.3 mg/dl, Conjugated bilirubin—2.0 mg/dl, Albumin $5.0 \mathrm{~g} / \mathrm{dl}$, while urinalysis was positive for bilirubin and excess urobilinogen, with no proteinuria. Pregnancy test was positive with estimated gestational age (EGA) of six weeks.

Abdominal ultrasonography showed bilaterally enlarged kidneys, right kidney measured $138 \mathrm{~mm}$ by $55 \mathrm{~mm}$, while the left kidney $159 \mathrm{~mm}$ by $63 \mathrm{~mm}$ ). Liver was enlarged (169 mm cranio-caudally) with uniform parenchyma echogenicity. The spleen was enlarged at $143 \mathrm{~mm}$ with homogenous parenchyma echo texture. Gallbladder contained multiple mobile echogenic foci in the dependent portion, casting posterior acoustic shadow; gallbladder thickness was normal.

She was managed as a case of acute cholecystolithiasis with viral Hepatitis B in early pregnancy.

She improved on intravenous fluids and antibiotics (IV ceftriaxone and metronidazole which was changed to oral cefuroxime and metronidazole), and Livolin forte (phosphatidylcholine), and was discharged after 6 days of admission for follow up in the out-patient department.

Repeat LFTs a week after the first one showed a downward trend in the parameters: AST 46 iu/l, ALT 54 iu/l, ALP $88 \mathrm{iu} / \mathrm{l}$, Total bilirubin—1.3 g/dl, Conjugated bilirubin—0.7 mg/dl, Albumin $4.8 \mathrm{~g} / \mathrm{dl}$.

\section{Discussion}

Given the multiplicity of aetiopathogenetic factors of hepatobiliary diseases in SCA, it is pertinent to determine the dominant cause of jaundice or liver dysfunction in patients with sickle hepatopathy, so as to focus on the clinical area needing immediate intervention and therefore effective targeted therapy. This requires adroit clinical skills to navigate the maze of clinical presentations and identify the offender among the culprits, thus preventing therapeutic misadventure.

The case presented would require some clinical sifting to really know the primal cause of her clinical presentation. Pregnancy is well known to present with some degree of haemodilution and reduction in some liver function parameters such as hypoproteinaemia and hypoalbuminaemia, whereas alkaline phosphatase tends to increase due to production in the placenta and bones of the fetus. Generally, ALT, AST, bilirubin and gamma glutamyl transferase tend to be normal throughout pregnancy but some clinical features of chronic liver disease such as telangiectasia or spider angiomas and palmar erythema may accompany normal pregnancy due to the hyperoestrogenic state [8]. Similarly, gall bladder motility is decreased during pregnancy, thus increasing the lithogenicity of the bile [9].

Our index patient was in early pregnancy (EGA 6 weeks), therefore physiological haemodilution is unlikely to explain most of her presentation. The raised ALT definitely suggests hepatocellular necrosis as a result of chronic hepatitis B, in view of the negative HBeAg and IgM anti-HBc and positive anti-HBe. She most likely had been exposed to hepatitis B infection during the multiple blood transfusion episodes, though horizontal transmission or vertical transmission could not be ruled out in this case. It is pertinent to know that in Nigeria, and in fact most of sub-Saharan Africa, where the sero-prevalence rate of HBsAg is about twenty percent, horizontal transmission is the main mode of transmission [10]. Also, the ALT-AST ratio of greater than two would further strengthen viral hepatitis as the cause of her chronic hepatitis, as she did not imbibe alcohol, and the ALT-AST ratio, which is usually less than one was not the case in her. Her conjugated bilirubin was greater than 20 percent suggesting conjugated hyperbilirubinaemia, however in view of the excess urobilinogen in the urine, there is a component of unconjugated hyperbilirubinaemia, thus presenting a dimorphic picture of hyperbilirubinaemia. The unconjugated aspect of the serum bilirubin was most likely due to haemolysis from the sickle cell disease and or haemolysis as a result of sepsis from the acute cholecystolithiasis.

Chronic cholecystolithiasis was safely ruled out because of the absence of thickening of the gall-bladder, a hallmark ultrasonographic finding in chronic cholecystitis. The gallstones found in her were also free and nonobstructive also making the possibility of cholestasis unlikely. The gall-bladder infection may also have been partly responsible for the tender hepatomegaly, which may also have been due to peri-hepatitis around the inflamed gall-bladder. The presence of bilirubinuria usually suggests parenchymal liver disease, especially in the absence of albuminuria. The absence of proteinuria in this patient also further strengthens the considered diagnosis of chronic hepatitis $B$ in the patient.

The well-being of the fetus in this patient should also be of paramount concern to the care-giver as the risk of intra-uterine and peri-natal transmission of HBV infection is real, though much less in this case because of the negative HBeAg and HBV-DNA viral load of less than $2000 \mathrm{iu} / \mathrm{l}$. It will be good and standard practice to ensure 
administration of both hepatitis B immunoglobulin and HBV vaccine within seventy two hours of birth, and to re-evaluate the patient for anti-hepatitis B therapy post-partum. The contacts of the patient would also require screening for HBV infection and subsequent immunization, if found negative.

\section{Conclusion}

It could therefore be concluded that our patient had background chronic hepatitis B with a superadded acute cholecystitis with non-obstructing gallstones, and possible subsequent bacterial hepatitis, which responded to antibiotics. The most likely dominant cause of jaundice in the patient was considered to be chronic hepatitis B that was precipitated by an infective process, in this case, acute cholecystitis. As the patient is being followed up at our out-patient department, it is expected that with close monitoring, the prognosis is good for the patient and the fetus, in spite of the risk of complications to the fetus and the mother.

\section{Acknowledgements}

We acknowledge the staff of the Chemical pathology and Radiology department for their contribution to investigating the patient.

\section{Conflict of Interest}

None declared.

\section{References}

[1] http://www.afro.who.int/en/nigeria/nigeria-publications/1775-.sickle\%20cell\%20disease.html

[2] Banerjee, S., Owen, C. and Chopra, S. (2001) Sickle Cell Hepatopathy: Concise Review. Hepatology, 33, $1021-1028$. http://dx.doi.org/10.1053/jhep.2001.24114

[3] Oladokun, A., Otegbayo, J.A. and Adeniyi, A.A. (2007) Viral Hepatitis in the Aetiogenesis of Jaundice in Pregnancy at the University College Hospital, Ibadan. African Journal of Medicine and Medical Sciences, 36, 115-118.

[4] Oladokun, A., Otegbayo, J.A. and Adeniyi, A.A. (2009) Maternal and Fetal Outcomes of Jaundice in Pregnancy at the University College Hospital, Ibadan. Nigerian Journal of Clinical Practice, 12, 277-280.

[5] Olaniyi, J.A. and Abjah, U.M. (2007) Frequency of Hepatomegaly and Splenomegaly in Nigerian Patients with Sickle Cell Disease. West African Journal of Medicine, 26, 274-277.

[6] Olaniyi, J.A. (2008) Multiple Complications in a Sickle Cell Disease Patient: A Case Report. Clinical Medicine Insights: Case Reports, 1, 97-100.

[7] Aken'ova, Y.A., Olasode, B.J., Ogunbiyi, J.O. and Thomas, J.O. (1993) Hepatobiliary Changes in Nigerians with Sickle Cell Anaemia. Annals of Tropical Medicine and Parasitology, 87, 603-606.

[8] Bacq, Y., Zarka, O., Brechot, J.F., et al. (1996) Liver Function Tests in Normal Pregnancies: A Prospective Study of 103 Pregnant Women and 103 Match Controls. Hepatology, 23, 1030-1034. http://dx.doi.org/10.1002/hep.510230514

[9] Lee, N.M. and Brady, C.W. (2009) Liver Disease in Pregnancy. World Journal of Gastroenterology, 15, 897-906. http://dx.doi.org/10.3748/wjg.15.897

[10] Botha, J.F., Ritchie, M.J., Dusheiko, G.M., Mouton, H.W. and Kew, M.C. (1984) Hepatitis B Virus Carrier State in Black Children in Ovamboland: Role of Perinatal and Horizontal Infection. The Lancet, 323, 1210-1212. http://dx.doi.org/10.1016/S0140-6736(84)91694-5 